\title{
Scaling, Multiscaling, and Nontrivial Exponents in Inelastic Collision Processes
}

\author{
E. Ben-Naim ${ }^{1}$ and P. L. Krapivsky ${ }^{2}$ \\ ${ }^{1}$ Theoretical Division and Center for Nonlinear Studies, Los Alamos National Laboratory, Los Alamos, New Mexico 87545 \\ ${ }^{2}$ Center for Polymer Studies and Department of Physics, Boston University, Boston, Massachusetts 02215
}

\begin{abstract}
We investigate velocity statistics of homogeneous inelastic gases using the Boltzmann equation. Employing an approximate uniform collision rate, we obtain analytic results valid in arbitrary dimension. In the freely evolving case, the velocity distribution is characterized by an algebraic large velocity tail, $P(v, t) \sim v^{-\sigma}$. The exponent $\sigma(d, \epsilon)$, a nontrivial root of an integral equation, varies continuously with the spatial dimension, $d$, and the dissipation coefficient, $\epsilon$. Although the velocity distribution follows a scaling form, its moments exhibit multiscaling asymptotic behavior. Furthermore, the velocity autocorrelation function decays algebraically with time, $A(t)=\langle\mathbf{v}(0) \cdot \mathbf{v}(t)\rangle \sim t^{-\alpha}$, with a non-universal dissipation-dependent exponent $\alpha=1 / \epsilon$. In the forced case, the steady state Fourier transform is obtained via a cumulant expansion. Even in this case, velocity correlations develop and the velocity distribution is non-Maxwellian.

PACS: 05.20.Dd, 02.50.-r, 47.70.Nd, 45.70.Mg
\end{abstract}

\section{INTRODUCTION}

Inelastic gases consist of hard sphere particles that interact via contact interactions and dissipate kinetic energy upon collisions [1]. They are used extensively to study dynamics of granular materials. Numerically, molecular dynamics simulations are quite successful in modeling many of the observed collective phenomena that include size segregation, phase transitions, shocks, clustering, and development of other spatial structures [2-10]. In parallel, kinetic theory is utilized to systematically derive macroscopic properties from the microscopic collision dynamics 11 13.

Inelastic gases, a prototype nonequilibrium interacting particle system, are interesting on their own rights 114 21. Recent theoretical and experimental studies show that the velocity distributions exhibit anomalous large-velocity statistics with exponential, stretched exponential, and Gaussian tails [6 8,22 25]. Inelastic gases involve significant velocity and spatial correlations in contrast with traditional molecular gases 2628 . Kinetic theory assumes that spatial velocity correlations are small. While this assumption can be justified for strongly driven gases, the situation for freely evolving gases is more difficult since velocity correlations can be ignored only in the early homogeneous phase [29 31], but must be taken into account in the asymptotic clustering phase. Clearly, the strong energy dissipation raises challenging new questions 32].

Yet, even more elementary questions remain unanswered. For example, random collision processes effectively generate thermal, purely Maxwellian, velocity distributions when the collisions are elastic. In particular, different components of the velocity become uncorrelated. In this study, we consider these very same processes but with inelastic collisions. We show that energy dissipation fundamentally alters the behavior. The system is intrinsically a nonequilibrium one, and the resulting velocity distributions are far from thermal.

We consider a collision process where random pairs of particles undergo inelastic collisions with a random impact direction. This process, often called the Maxwell model, is described by a Boltzmann equation with a uniform collision rate. In classical kinetic theory of gases, the analytically tractable Maxwell model precedes the Boltzmann equation [33]. Historically, it played an important role in the development of kinetic theory [34 36], and it still remains the subject of current research [37, 38].

Very recently, it has been noted that the Maxwell model is analytically tractable even for inelastic collisions [39 43]. Interesting behavior emerges in the freely evolving case. In one dimension, while moments of the velocity distribution exhibit multiscaling [39], the velocity distribution itself still approaches a scaling form with an algebraic large velocity tail 40]. Here, we show analytically that in arbitrary spatial dimension the velocity distribution admits a scaling solution with an algebraic large velocity tail. The corresponding exponent, a root of a transcendental equation, depends on the spatial dimension and the restitution coefficient. Additionally, we find that the multiscaling behavior extends to higher dimensions, and that the velocity autocorrelation function exhibits aging and nonuniversal asymptotic behavior. In general, velocity components develop significant correlations. Such correlations diminish in the forced case, although the velocity distribution remains non-Maxwellian.

The rest of this paper is organized as follows. The basic Boltzmann equation for the velocity distribution and its Fourier transform are presented in Sec. II. In Sec. III, we investigate the scaling regime, and obtain the extremal velocity statistics, moments of the velocity scaling function, and velocity correlations. In Sec. IV, we illuminate the nonequilibrium dynamics by studying the time dependent behavior of the moments and the velocity autocorrelation function. In Sec. V, we consider nonequilibrium steady states in the driven case, and obtain the steady state distribution as a cumulant expansion. A few generalizations are briefly mentioned in Sec. VI, and conclusions are given in Sec. VII. 


\section{THE MAXWELL MODEL}

We study a homogeneous system of identical inelastic spherical particles. The mass and the cross-section are set to unity without loss of generality. Particles interact via binary collisions that lead to exchange of momentum along the impact direction. The post-collision velocities $\mathbf{v}_{1,2}$ are given by a linear combination of the pre-collision velocities $\mathbf{u}_{1,2}$,

$$
\mathbf{v}_{1,2}=\mathbf{u}_{1,2} \mp(1-\epsilon)(\mathbf{g} \cdot \mathbf{n}) \mathbf{n} .
$$

Here $\mathbf{g}=\mathbf{u}_{1}-\mathbf{u}_{2}$ is the relative velocity and $\mathbf{n}$ the unit vector connecting the particles' centers. In each collision, the normal component of the relative velocity is reduced by the restitution coefficient $r=1-2 \epsilon$. The energy dissipation equals $\Delta E=-\epsilon(1-\epsilon)(\mathbf{g} \cdot \mathbf{n})^{2}$, so for $\epsilon=0$ collisions are elastic, while for $\epsilon=1 / 2$ collisions are perfectly inelastic with maximal energy dissipation. Since the collision rule (1) is Galilean invariant, the average velocity can be set to zero without loss of generality.

We investigate the "Maxwell model" where the collision rate in the Boltzmann equation equals the typical velocity, rather than the actual relative velocity [35,36. This kinetic theory describes a stochastic process where randomly chosen pairs of particles undergo inelastic collisions according to (1) with a randomly chosen impact direction $\mathbf{n}$. In such a process, no spatial correlations develop, and the normalized velocity distribution function, $P(\mathbf{v}, t)$, obeys

$$
\begin{aligned}
\frac{\partial P(\mathbf{v}, t)}{\partial t} & =g \int d \mathbf{n} \int d \mathbf{u}_{1} P\left(\mathbf{u}_{1}, t\right) \int d \mathbf{u}_{2} P\left(\mathbf{u}_{2}, t\right) \\
& \times\left\{\delta\left[\mathbf{v}-\mathbf{u}_{1}+(1-\epsilon)(\mathbf{g} \cdot \mathbf{n}) \mathbf{n}\right]-\delta\left(\mathbf{v}-\mathbf{u}_{1}\right)\right\}
\end{aligned}
$$

The overall collision rate equals $g=\sqrt{T}$ where $T$ is the granular temperature, or the average velocity fluctuation per degree of freedom, $T=\frac{1}{d} \int d \mathbf{v} v^{2} P(\mathbf{v}, t)$ with $v \equiv|\mathbf{v}|$. The restriction $\mathbf{g} \cdot \mathbf{n}>0$ on the angular integration range in Eq. (2) can be tacitly ignored, because the integrand obeys the reflection symmetry $\mathbf{n} \rightarrow-\mathbf{n}$. This angular integration should be normalized, $\int d \mathbf{n}=1$.

We study primarily the freely evolving case where in the absence of energy input the system "cools" indefinitely. From the Boltzmann equation (2), the temperature rate equation is

$$
\frac{d}{d t} T=-\lambda T^{3 / 2}, \quad \text { with } \quad \lambda=\frac{2 \epsilon(1-\epsilon)}{d} .
$$

The constant $\lambda=2 \epsilon(1-\epsilon) \int d \mathbf{n} n_{1}^{2}$, is obtained using the identity $n_{1}^{2}+\ldots+n_{d}^{2}=1$ that yields $\int d \mathbf{n} n_{1}^{2}=1 / d$. Solving Eq. (3) we find that the temperature decays according to Haff's cooling law [14]

$$
T(t)=T_{0}\left(1+t / t_{*}\right)^{-2},
$$

with the time scale $t_{*}=d /\left[\epsilon(1-\epsilon) \sqrt{T_{0}}\right]$ set by the initial temperature, $T_{0}$.
Given the convolution structure of the Boltzmann equation (2), we introduce the Fourier transform 36] of the velocity distribution function,

$$
F(\mathbf{k}, t)=\int d \mathbf{v} e^{i \mathbf{k} \cdot \mathbf{v}} P(\mathbf{v}, t) .
$$

We conveniently reset the collision rate to unity by modifying the time variable. The collision counter $\tau$ is defined via the transformation $\frac{d}{d \tau}=\frac{1}{\sqrt{T}} \frac{d}{d t}$. Specifically,

$$
\tau=\frac{2}{\lambda} \ln \left(1+t / t_{*}\right)
$$

equals the average number of collisions experienced by a particle. Applying the Fourier transform to Eq. (2) and integrating over the velocities gives

$$
\frac{\partial}{\partial \tau} F(\mathbf{k}, \tau)+F(\mathbf{k}, \tau)=\int d \mathbf{n} F[\mathbf{k}-\mathbf{q}, \tau] F[\mathbf{q}, \tau]
$$

with $\mathbf{q}=(1-\epsilon)(\mathbf{k} \cdot \mathbf{n}) \mathbf{n}$. This equation reflects the momentum transfer occurring during collisions.

We restrict our attention to isotropic situations, and write the Fourier transform $F(\mathbf{k}, \tau) \equiv F(z, \tau)$ in terms of the variable $z=k^{2}$. To perform the angular integration, it proves useful to employ spherical coordinates with the polar axis parallel to $\mathbf{k}$, so that $\hat{\mathbf{k}} \cdot \mathbf{n}=\cos \theta$. The $\theta$ dependent factor of the measure $d \mathbf{n}$ is proportional to $(\sin \theta)^{d-2} d \theta$. In terms of the variable $\mu=\cos ^{2} \theta$ one has $d \mathbf{n} \equiv \mathcal{D} \mu$, with

$$
B\left(\frac{1}{2}, \frac{d-1}{2}\right) \mathcal{D} \mu=\mu^{-\frac{1}{2}}(1-\mu)^{\frac{d-3}{2}} d \mu
$$

where $B(a, b)$ is the beta function. This integration measure is properly normalized, $\int_{0}^{1} \mathcal{D} \mu=1$. Hereinafter, we denote angular integration with brackets

$$
\langle f\rangle=\int_{0}^{1} \mathcal{D} \mu f(\mu)
$$

The governing equation (7) for the Fourier transform can now be rewritten in the convenient from

$$
\frac{\partial}{\partial \tau} F(z, \tau)+F(z, \tau)=\langle F(\xi z, \tau) F(\eta z, \tau)\rangle,
$$

with the shorthand notations $\xi=1-\left(1-\epsilon^{2}\right) \mu$ and $\eta=(1-\epsilon)^{2} \mu$. Hence, the Fourier equation is both nonlinear and non-local. Interestingly, while it is difficult to integrate this equation with respect to time, most of the physically relevant features of the velocity distributions including large velocity statistics and the time dependent behavior of the moments can be found analytically, as will be shown below. 


\section{SCALING SOLUTIONS}

Numerical simulations in two-dimensions suggest that the velocity distribution approaches the scaling form 40]

$$
P(\mathbf{v}, t) \sim \frac{1}{T^{d / 2}} \mathcal{P}\left(\frac{v}{\sqrt{T}}\right) .
$$

The scaling form of the Fourier transform reads

$$
F(\mathbf{k}, t)=\Phi(x), \quad \text { with } \quad x=k^{2} T .
$$

In the $k \rightarrow 0$ limit, the Fourier transform behaves as $F(\mathbf{k}, t) \cong 1-\frac{1}{2} k^{2} T$. This implies that the first two terms in the Taylor expansion of the corresponding scaling function are universal, $\Phi(x) \cong 1-\frac{1}{2} x$. Substituting the above scaling form into the governing equation (10) and using the temperature cooling rate $\frac{d}{d \tau} T=-\lambda T$ yields the governing equation for the scaling function

$$
-\lambda x \Phi^{\prime}(x)+\Phi(x)=\langle\Phi(\xi x) \Phi(\eta x)\rangle .
$$

One can check that the velocity distribution is purely Maxwellian $\Phi(x)=e^{-x / 2}$ in the elastic case 44. Indeed, $\lambda=0$ and $\xi+\eta=1$ in this case. A stochastic process of elastic collisions effectively randomizes the velocities and leads to a thermal distribution.

\section{A. Algebraic tails}

It is instructive to consider first the one-dimensional case. Here, integration over $\mu$ is immediate as this variable equals unity, and the scaling function satisfies $-\lambda x \Phi^{\prime}(x)+\Phi(x)=\Phi\left[\epsilon^{2} x\right] \Phi\left[(1-\epsilon)^{2} x\right]$. Remarkably, this non-local non-linear differential equation admits a very simple solution 40]

$$
\Phi(x)=(1+\sqrt{x}) e^{-\sqrt{x}} .
$$

Performing the inverse Fourier transform gives the velocity distribution as a squared Lorentzian

$$
\mathcal{P}(w)=\frac{2}{\pi}\left(1+w^{2}\right)^{-2} .
$$

The scaling solution (15) is universal as it is independent of the dissipation coefficient $\epsilon$. Its key feature is the algebraic tail, $\mathcal{P}(w) \sim w^{-4}$ as $|w| \rightarrow \infty$.

In general dimension $d$, the large velocity behavior of the velocity distribution can be determined from the small wave number behavior of its Fourier transform. For example, the small- $x$ expansion of the one-dimensional solution (14) contains both regular and singular terms: $\Phi(x)=1-\frac{1}{2} x+\frac{1}{3} x^{3 / 2}+\cdots$, and the dominant singular $x^{3 / 2}$ term reflects the $w^{-4}$ tail of $\mathcal{P}(w)$. In general, an algebraic tail of the velocity distribution (11),

$$
\mathcal{P}(w) \sim w^{-\sigma} \quad \text { as } \quad w \rightarrow \infty
$$

indicates the existence of a singular component in the Fourier transform,

$$
\Phi_{\text {sing }}(x) \sim x^{(\sigma-d) / 2} \quad \text { as } \quad x \rightarrow 0 .
$$

The inverse is also correct. This can be seen by recasting the Fourier transform $\Phi(x) \propto \int_{0}^{\infty} d w w^{d-1} \mathcal{P}(w) e^{i w \sqrt{x}}$ into a Laplace transform $I(s) \propto \int_{0}^{\infty} d w w^{d-1} \mathcal{P}(w) e^{-w s}$ by writing $x=-s^{2}$. The small-s expansion of $I(s)$ contains regular and singular components. For example, when $\sigma<d$, the integral $I(s)$ diverges as $s \rightarrow 0$ and integration over large- $w$ yields the dominant contribution $I_{\text {sing }}(s) \sim s^{\sigma-d}$. When $d<\sigma<d+1, I(0)$ is finite, but the next term is the above singular term, so $I(s)=I(0)+I_{\text {sing }}(s)+\cdots$. In general, the singular contribution is $I_{\text {sing }}(s) \sim s^{\sigma-d}$, thereby leading to Eq. (17).

The exponent $\sigma$ can be now obtained by inserting $\Phi(x)=\Phi_{\text {reg }}(x)+\Phi_{\text {sing }}(x)$ into Eq. (13) and balancing the dominant singular terms. We find that $\sigma$ is a root of the integral equation

$$
1-\lambda \frac{\sigma-d}{2}=\left\langle\xi^{(\sigma-d) / 2}+\eta^{(\sigma-d) / 2}\right\rangle .
$$

This relation, originally derived in Refs. [41,42], can be recast as an eigenvalue problem. Indeed, defining $\lambda_{\nu}=\left\langle 1-\xi^{\nu}-\eta^{\nu}\right\rangle$ we can re-write Eq. (18) as $\lambda_{\mu}=\mu \lambda_{1}$ with $\mu=\frac{\sigma-d}{2}$. Note that $\lambda \equiv \lambda_{1}$, so there is an obvious solution $\mu=1$, or $\sigma=d+2$. In this case, the singular term simply coincides with the dominant regular term, $x^{(\sigma-d) / 2}=x$. Hence, this solution is trivial and in the following we shall seek a solution with $\sigma>d+2$.

The integral equation (18) can also be rewritten in terms of special functions. The first integral on the righthand side of (18) can be expressed in terms of the hypergeometric function ${ }_{2} F_{1}(a, b ; c ; z)$ [45 and the second as a ratio of beta functions:

$$
\begin{aligned}
& 1-\epsilon(1-\epsilon) \frac{\sigma-d}{d}= \\
& { }_{2} F_{1}\left[\frac{d-\sigma}{2}, \frac{1}{2} ; \frac{d}{2} ; 1-\epsilon^{2}\right]+(1-\epsilon)^{\sigma-d} \frac{\Gamma\left(\frac{\sigma-d+1}{2}\right) \Gamma\left(\frac{d}{2}\right)}{\Gamma\left(\frac{\sigma}{2}\right) \Gamma\left(\frac{1}{2}\right)} .
\end{aligned}
$$

We conclude that the exponent $\sigma \equiv \sigma(d, \epsilon)$ depends in a nontrivial fashion on the spatial dimension $d$ as well as the dissipation coefficient $\epsilon$.

First, let us investigate the dependence on the dissipation coefficient by considering the quasi-elastic limit $\epsilon \rightarrow 0$. In the elastic case, the Maxwellian distribution, $\Phi(x)=e^{-x / 2}$, implies a diverging exponent $\sigma \rightarrow \infty$ as $\epsilon \rightarrow 0$. Therefore, the right-hand side of Eq. (19) vanishes in the quasi-elastic limit and to leading order

$$
\sigma \simeq \frac{d}{\epsilon}
$$

Clearly, the quasi-elastic limit is singular. Dissipation, even if minute, seriously changes the nature of the system [6, 25, 31]. Further corrections can be obtained via a 
systematic perturbation expansion in $\epsilon$. We merely quote the two leading corrections in the physically relevant dimensions

$$
\begin{aligned}
& \sigma(2, \epsilon)=\frac{2}{\epsilon}-\frac{2\left(e^{-2}+1\right)}{\sqrt{\pi \epsilon}}+\frac{4 \pi-\left(e^{-2}+1\right)^{2}}{\pi}+\mathcal{O}\left(\epsilon^{1 / 2}\right), \\
& \sigma(3, \epsilon)=\frac{3}{\epsilon}-\sqrt{\frac{3 \pi}{2 \epsilon}}+\left[6-e^{-3}-\frac{\pi}{4}\right]+\mathcal{O}\left(\epsilon^{1 / 2}\right) .
\end{aligned}
$$

Next, we discuss the dependence on the dimension. First, one can verify that $\sigma=4$ when $d=1$ by utilizing the identity ${ }_{2} F_{1}(a, b ; b ; z)=(1-z)^{-a}$. In the infinite dimension limit, the second integral $\left\langle\eta^{(\sigma-d) / 2}\right\rangle$ in Eq. (18) is negligible as it vanishes exponentially with the dimension. To evaluate the second integral we take the limits $d \rightarrow \infty$ and $\mu \rightarrow 0$ with $z=\mu d / 2$ fixed. The integration measure (8) is transformed according to $\mathcal{D} \mu \rightarrow(\pi z)^{-1 / 2} e^{-z} d z$, and the basic Eq. (18) becomes $1-\epsilon(1-\epsilon) u=\int_{0}^{\infty} d z(\pi z)^{-1 / 2} e^{-\left[1+\left(1-\epsilon^{2}\right) u\right] z}$ with the shorthand notation $u=\frac{\sigma}{d}-1$. Performing the integration yields $1-\epsilon(1-\epsilon) u=\left[1+\left(1-\epsilon^{2}\right) u\right]^{-1 / 2}$. This cubic equation has the aforementioned trivial solution $u=0$ and two non-trivial solutions. Choosing the physically relevant $u$, we obtain that as $d \rightarrow \infty$

$$
\frac{\sigma}{d}=\frac{1+\frac{3}{2} \epsilon-\epsilon^{3}-\epsilon^{1 / 2}\left(1+\frac{5}{4} \epsilon\right)^{1 / 2}}{\epsilon\left(1-\epsilon^{2}\right)} .
$$

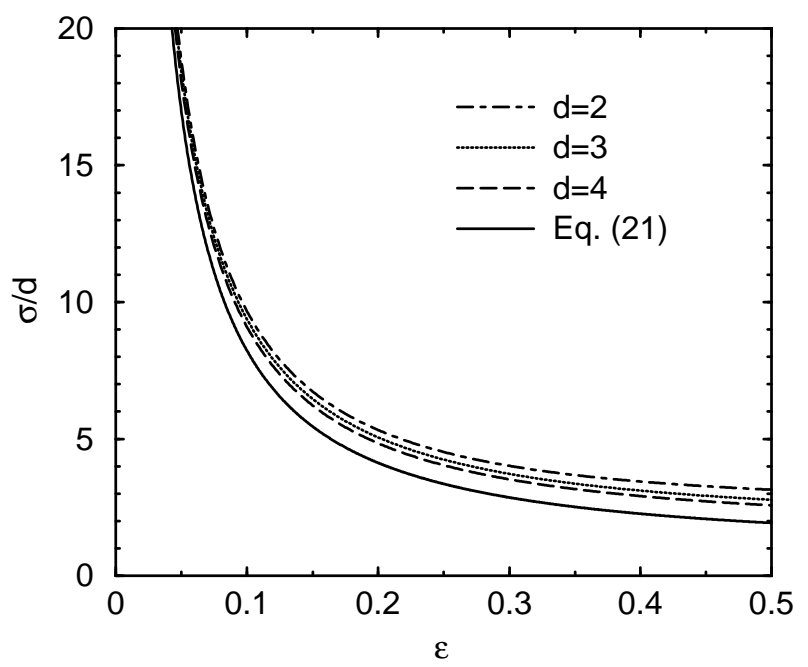

FIG. 1. The exact exponent $\sigma$, obtained from Eq. (19), versus the dissipation parameter $\epsilon$. The exponent was scaled by the dimension $d$. Shown also is the limiting large dimension expression (21).

In general, $\sigma \propto d$, and therefore, the algebraic decay becomes sharper as the dimension increases. The exponent $\sigma(d, \epsilon)$ increases monotonically with increasing $d$, and additionally, it increases monotonically with decreasing $\epsilon$ (see Fig. 1). Both features are intuitive as they mirror the monotonic dependence of the energy dissipation rate $\lambda=2 \epsilon(1-\epsilon) / d$ on $d$ and $\epsilon$. Hence, the com- pletely inelastic case provides a lower bound for the exponent, $\sigma(d, \epsilon) \geq \sigma(d, \epsilon=1 / 2)$ with $\sigma(d, 1 / 2)=6.28753$, 8.32937 , for $d=2,3$, respectively. Numerical simulation results are consistent with the former value [46]. The algebraic tails are characterized by unusually large exponents which may be difficult to measure accurately in practice; for instance, typical granular particles are characterized by the dissipation coefficient $\epsilon \approx 0.1$ yielding $\sigma \approx 30$ in three dimensions. Figure 1 also shows that the quantity $\sigma / d$ weakly depends upon the dimension, and the large- $d$ limit (21) provides a good approximation even at moderate dimensions.

\section{B. Divergence of the moments}

The algebraic tail of the velocity distribution implies that sufficiently small moments of the scaling function $\Phi(x)$ are finite, while moments larger than some index diverge. In the scaling regime, moments of the velocity distribution can be calculated by expanding the Fourier transform in powers of $x$,

$$
\Phi(x)=\sum_{n \geq 0} \phi_{n}(-x)^{n} .
$$

The coefficients $\phi_{n}$ yield the leading asymptotic behavior of the velocity moments, $M_{k}(t)=\int d \mathbf{v} v^{k} P(\mathbf{v}, t)$, via the relation $(2 n) ! T^{n} \phi_{n} \simeq\left\langle\mu^{n}\right\rangle M_{2 n}$. Inserting the moment expansion into the governing equation (13) yields the closed hierarchy of equations

$$
\left(\lambda_{n}-n \lambda_{1}\right) \phi_{n}=\sum_{m=1}^{n-1} \lambda_{m, n-m} \phi_{m} \phi_{n-m}
$$

with $\lambda_{n}=\left\langle 1-\xi^{n}-\eta^{n}\right\rangle$ and $\lambda_{m, l}=\left\langle\xi^{m} \eta^{l}\right\rangle$. The first few coefficients are written explicitly in Appendix A. Starting with $\phi_{0}=1$ and $\phi_{1}=1 / 2$, further coefficients are determined recursively from (23). In the elastic case $(\epsilon=0)$, one has $\phi_{n}=\left(n ! 2^{n}\right)^{-1}$, consistent with $\Phi(x)=e^{-x / 2}$. For general $\epsilon$, the first two terms are

$$
\begin{aligned}
& \phi_{2}=\frac{1}{8} \frac{1-3 \frac{1-\epsilon^{2}}{d+2}}{1-3 \frac{1+\epsilon^{2}}{d+2}}, \\
& \phi_{3}=\frac{1}{48} \frac{1-3 \frac{1-\epsilon^{2}}{d+2}}{1-3 \frac{1+\epsilon^{2}}{d+2}} \frac{1-3 \frac{(1-\epsilon)(1+3 \epsilon)}{d+2}+30 \frac{\epsilon(1-\epsilon)\left(1-\epsilon^{2}\right)}{(d+2)(d+4)}}{1-3 \frac{(1+\epsilon)^{2}}{d+2}+10 \frac{\epsilon(1-\epsilon)\left(3+\epsilon^{2}\right)}{(d+2)(d+4)}} .
\end{aligned}
$$

The behavior is determined by two parameters: $d$ and $\epsilon$. Fixing $\epsilon$, we see that a given moment $\phi_{n}$ is finite only if the dimension is sufficiently large, $d>d_{n}(\epsilon)$. In particular, $\phi_{n}$ is finite only if the left-hand side of Eq. (23) is positive, $\lambda_{n}-n \lambda_{1}>0$. This condition is satisfied only if the dimension is sufficiently large $d>d_{n}$, with $d_{n}$ being the spatial dimension at which $\lambda_{n}=n \lambda_{1}$. For example, $\phi_{2}>0$ when $d>d_{2}$, and $\phi_{3}$ is finite only when $d>d_{3}$ with the following crossover dimensions 
$d_{2}=1+3 \epsilon^{2}$

$d_{3}=\frac{3}{2}\left(\epsilon^{2}+2 \epsilon-1\right)+\frac{1}{2} \sqrt{25-60 \epsilon+186 \epsilon^{2}-4 \epsilon^{3}+49 \epsilon^{4}}$.

Conversely, for a fixed dimension, a given moment is finite only if the dissipation is sufficiently small. For example, $\phi_{3}$ is positive only when $\epsilon<0.302074,0.427438$ at $d=2,3$. Such values, obtained by solving polynomial equations yield integer values of the large velocity decay exponent $\sigma(2,0.302074)=8$ and $\sigma(3,0.427438)=9$, in accord with direct numerical solution of Eq. (19).

\section{Velocity correlations}

Maxwell's seminal derivation of the Maxwellian distribution (see Ref. [26], p. 36) relies on two basic assumptions: (1) Isotropy of the velocity distribution, and (2) Absence of correlations between the velocity components. The latter assumption is directly probed using the following correlation measure

$$
Q=\frac{\left\langle v_{x}^{2} v_{y}^{2}\right\rangle-\left\langle v_{x}^{2}\right\rangle\left\langle v_{y}^{2}\right\rangle}{\left\langle v_{x}^{2}\right\rangle\left\langle v_{y}^{2}\right\rangle}
$$

A non-vanishing $Q$ indicates that velocity correlations do exist, and the larger $Q$ the larger the correlation. In the freely evolving case, this quantity easily follows from the small- $x$ behavior of the scaling function $\Phi(x)$. By definition, $\left\langle v_{x}^{2}\right\rangle=\left\langle v_{y}^{2}\right\rangle=T$ and furthermore, $\left\langle v_{x}^{2} v_{y}^{2}\right\rangle=\left.\frac{\partial^{2}}{\partial k_{x}^{2}} \frac{\partial^{2}}{\partial k_{y}^{2}} F\right|_{\mathbf{k}=0}=4 T^{2} \Phi^{\prime \prime}(0)$. Consequently, one has $Q=4 \Phi^{\prime \prime}(0)-1=8 \phi_{2}-1$. Using Eq. (24) we find

$$
Q=\frac{6 \epsilon^{2}}{d-\left(1+3 \epsilon^{2}\right)}
$$

when $d>d_{2}=1+3 \epsilon^{2}$, and $Q=\infty$ otherwise. While the quantity $Q$ is physical when $d \geq 2$, it is sensible to use analytic continuation to reveal the underlying divergence. Velocity correlations vanish for elastic gases. Interestingly, inelasticity introduces strong velocity correlations, and the larger $\epsilon$ the larger the correlations as $Q$ increases monotonically with increasing $\epsilon$. The perfectly inelastic case $(\epsilon=1 / 2)$ again provides a bound: $Q<Q_{\max }=6$, $6 / 5$ for $d=2,3$, respectively. This behavior is somewhat intuitive as the unisotropic collision rule (11) discriminates the velocity component normal to the impact direction.

\section{NONEQUILIBRIUM DYNAMICS}

Thus far, we focused on the leading asymptotic behavior of the velocity distribution. The diverging moments and the dissipative nature of this system suggest that the time dependence may exhibit rich behavior. Thus, we study relaxation of velocity characteristics such as the moments and the autocorrelation function.

\section{A. Multiscaling of the moments}

While moments of the scaling function diverge, the actual moments must remain finite at all times, particularly at the scaling regime. Therefore, the above moment analysis suggests that knowledge of the leading asymptotic behavior is not sufficient to characterize the time dependent behavior of sufficiently large moments.

The time evolution of the moments can be studied using the expansion

$$
F(z, \tau)=\sum_{n=0}^{\infty} f_{n}(\tau)(-z)^{n}
$$

The actual moments are related to the coefficients via $(2 n) ! f_{n}=\left\langle\mu^{n}\right\rangle M_{2 n}$. Substituting the expansion (28) into (10) yields the evolution equations

$$
\frac{d}{d \tau} f_{n}+\lambda_{n} f_{n}=\sum_{m=1}^{n-1} \lambda_{m, n-m} f_{m} f_{n-m}
$$

We demonstrate multiscaling asymptotic behavior by evaluating the second, fourth, and sixth moments. The second moment is obtained from $\frac{d}{d \tau} f_{1}+\lambda_{1} f_{1}=0$ with $\lambda_{1}=\lambda=2 \epsilon(1-\epsilon) / d$. Hence, we recover Haff's law $f_{1}(\tau)=f_{1}(0) e^{-\lambda_{1} \tau}$, or $f_{1}(t)=f_{1}(0)\left(1+t / t_{*}\right)^{-2}$. Asymptotically, the second moment of the velocity distribution has the universal behavior, $M_{2} \sim t^{-2}$. The next coefficient $f_{2}$ satisfies

$$
\frac{d}{d \tau} f_{2}+\lambda_{2} f_{2}=\lambda_{1,1} f_{1}^{2} .
$$

Solving Eq. (30) we find that $f_{2}(\tau)$ is a linear combination of two exponentials, $e^{-\lambda_{2} \tau}$ and $e^{-2 \lambda_{1} \tau}$, whose decay coefficients are equal $\lambda_{2}=2 \lambda_{1}$ at the crossover dimension $d_{2}$. Integrating the rate equation (30) and translating back to the physical time $t$, we obtain

$$
f_{2}(t)=C_{1}\left(1+t / t_{*}\right)^{-4}+C_{2}\left(1+t / t_{*}\right)^{-2 \alpha_{2}}
$$

for $d \neq d_{2}$. Here, $\alpha_{n}=\lambda_{n} / \lambda_{1}, C_{1}=\lambda_{1,1} f_{1}^{2}(0) /\left(\lambda_{2}-2 \lambda_{1}\right)$, and $C_{2}=f_{2}(0)-C_{1}$. When $d=d_{2}$ one finds

$$
f_{2}(t)=\left[C_{1} \ln \left(1+t / t_{*}\right)+C_{2}\right]\left(1+t / t_{*}\right)^{-4},
$$

with $C_{1}=\lambda_{1,1} f_{1}^{2}(0)$ and $C_{2}=f_{2}(0)$. Thus for $d>d_{2}$, the fourth moment exhibits ordinary scaling, $f_{2} \sim t^{-4}$, or $M_{4} \sim M_{2}^{2} \sim t^{-4}$. When $d<d_{2}$, multiscaling becomes apparent as $f_{2} \sim t^{-2 \alpha_{2}}$ and therefore the ratio $M_{4} / M_{2}^{2}$ diverges asymptotically. This is consistent with the divergence of the fourth moment of the scaling function $\Phi(x)$ that occurs at the same crossover dimension $d_{2}$. A logarithmic correction occurs at this dimension. In summary, we find the following leading asymptotic behavior of the fourth moment 


$$
M_{4}(t) \sim \begin{cases}t^{-4} & d>d_{2}, \\ t^{-4} \ln t & d=d_{2}, \\ t^{-2 \alpha_{2}} & d<d_{2} .\end{cases}
$$

A similar calculation can be carried for the sixth moment. The solution of $\frac{d}{d \tau} f_{3}+\lambda_{3} f_{3}=\left(\lambda_{1,2}+\lambda_{2,1}\right) f_{1} f_{2}$, with $f_{1}$ and $f_{2}$ given above involves three exponentials: $e^{-\lambda_{3} \tau}, e^{-\left(\lambda_{1}+\lambda_{2}\right) \tau}$, and $e^{-3 \lambda_{1} \tau}$. Asymptotically, the first exponential dominates when $d<d_{3}$, and consequently, $M_{6} \sim t^{-2 \alpha_{3}}$; otherwise, the third exponential dominates and thence ordinary scaling occurs, $M_{6} \sim t^{-6}$. Generally, the leading asymptotic behavior of the $2 n$-th moment is characterized by two different regimes

$$
M_{2 n}(t) \sim \begin{cases}t^{-2 n} & d>d_{n}, \\ t^{-2 n} \ln t & d=d_{n}, \\ t^{-2 \alpha_{n}} & d<d_{n} .\end{cases}
$$

Further logarithmic corrections affecting sub-dominant terms occur at the crossover dimensions $d_{2}, \ldots, d_{n-1}$.

The dependence of $d_{n}(\epsilon)$ on the dissipation coefficient is shown in Figure 2. In the physical dimensions $d=2,3$, the fourth moment exhibits ordinary scaling behavior. The sixth order moment exhibits multiscaling if the dissipation coefficient is large enough: $\epsilon>0.302074,0.427438$ for $d=2,3$, respectively. In the large $n$ limit, $\lambda_{n} \rightarrow 1$ so from $\lambda_{n}=n \lambda_{1}$ we find $d_{n} \rightarrow 2 \epsilon(1-\epsilon) n$. Thus, regardless of the dissipation parameters and the dimension, sufficiently large moments exhibit multiscaling:

$$
M_{2 n} \propto M_{2}^{\alpha_{n}}, \quad \alpha_{n}=\lambda_{n} / \lambda_{1}
$$

Interestingly, the multiscaling exponents saturate asymptotically, $\alpha_{n} \rightarrow d /[2 \epsilon(1-\epsilon)]$ as $n \rightarrow \infty$. Of course, if the dimension increases or the dissipation parameter decreases, the order of the lowest moment exhibiting multiscaling increases, and in practice, it may be difficult to observe deviations from ordinary scaling. For example, at $d=3$ and $\epsilon=0.1$, multiscaling occurs only for moments whose index exceeds 30 !

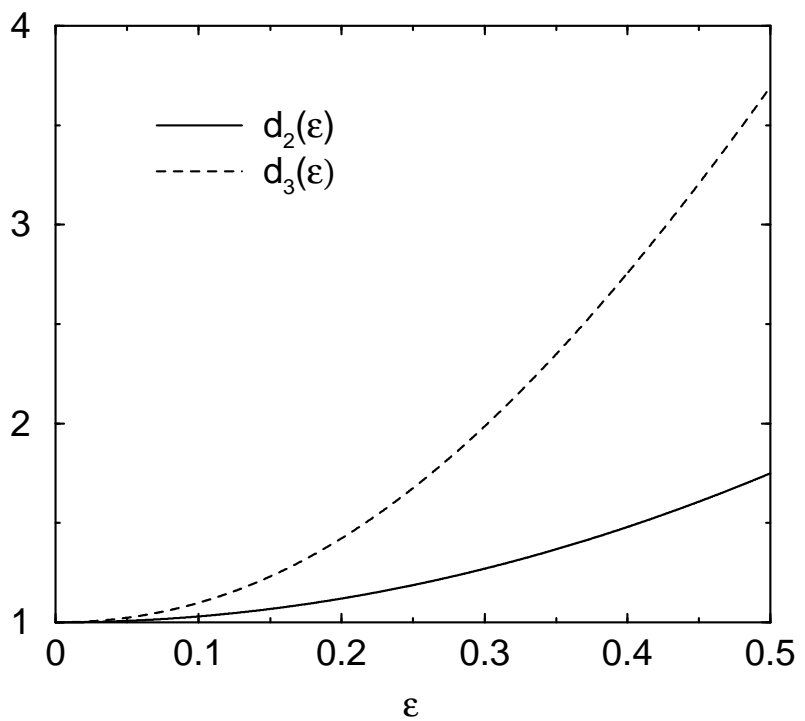

FIG. 2. The crossover dimensions $d_{n}(\epsilon)$ of Eq. 25) versus the dissipation coefficient for $n=2,3$.

\section{B. Non-universal velocity autocorrelations}

The autocorrelation function quantifies memory in the velocity of a tagged particle [26]. The velocity autocorrelation, $A\left(t_{w}, t\right)$, is defined via

$$
A\left(t_{w}, t\right)=\overline{\mathbf{v}\left(t_{w}\right) \cdot \mathbf{v}(t)}
$$

where the overline denotes averaging over all particles and $t_{w}$ is the "waiting" time, $t_{w}<t$.

It is simple to show (see appendix B) that the autocorrelation evolves according to the following linear equation

$$
\frac{d}{d \tau} A\left(\tau_{w}, \tau\right)=-\frac{1-\epsilon}{d} A\left(\tau_{w}, \tau\right)
$$

where time is again expressed in terms of the collision counters $\tau_{w}$ and $\tau$. Equation (37) is solved to give $A\left(\tau_{w}, \tau\right)=A\left(\tau_{w}, \tau_{w}\right) \exp \left[-\frac{1-\epsilon}{d}\left(\tau-\tau_{w}\right)\right]$, or equivalently

$$
A\left(t_{w}, t\right)=A_{0}\left(1+t_{w} / t_{*}\right)^{1 / \epsilon-2}\left(1+t / t_{*}\right)^{-1 / \epsilon},
$$

with $A_{0}=d T_{0}$. Therefore, $A\left(t_{w}, t\right)$ is a function of the waiting time $t_{w}$ and the observation time $t$, and not simply of their difference, $t-t_{w}$. This interesting history dependence or "aging" is another signature of the nonequilibrium nature of our system.

Memory of the initial conditions can be quantified by setting $t_{w}=0$. Writing $A(t) \equiv A(0, t)$ we arrive at the following algebraic decay

$$
A(t)=A_{0}\left(1+t / t_{*}\right)^{-1 / \epsilon} .
$$

In contrast with the temperature which decays with a universal law, $T(t) \sim t^{-2}$, the autocorrelation decays with a non-universal law, $A(t) \sim t^{-1 / \epsilon}$. The exponent is independent of the dimension. However, it strongly depends on the dissipation, and the stronger the dissipation, the stronger the memory of the initial conditions. This decay exponent is bounded by $2 \leq 1 / \epsilon \leq \infty$. In the elastic case, $\epsilon=0$, a simple exponential decay occurs, and in the totally inelastic case, $\epsilon=1 / 2$, the autocorrelation and the temperature are proportional to each other.

The autocorrelation function allows calculation of the long-time spread in the position of a tagged particle $\Delta^{2}(t) \equiv\left\langle|\mathbf{x}(t)-\mathbf{x}(0)|^{2}\right\rangle$. Using $\mathbf{x}(t)-\mathbf{x}(0)=\int_{0}^{t} d t^{\prime} \mathbf{v}\left(t^{\prime}\right)$, one can immediately express $\Delta^{2}(t)$ via the autocorrelation function, $\Delta^{2}=2 \int_{0}^{t} d t^{\prime} \int_{0}^{t^{\prime}} d t^{\prime \prime} A\left(t^{\prime \prime}, t^{\prime}\right)$. Substituting (38) into this expression and performing the integration yields $\Delta^{2}(t)=C_{1} \ln \left(1+t / t_{*}\right)+C_{2}\left[\left(1+t / t_{*}\right)^{1-1 / \epsilon}-1\right]$ with $C_{1}=2 A_{0} t_{*}^{2} \epsilon /(1-\epsilon)$ and $C_{2}=-C_{1} \epsilon /(1-\epsilon)$. Asymptotically, the second term is negligible, and the spread has a generic logarithmic behavior

$$
\Delta \sim \sqrt{\ln t}
$$

reflecting the $t^{-1}$ decay of the overall velocity scale 47, 48. 


\section{STEADY STATES}

Thus far, we have discussed freely cooling systems where the energy decreases indefinitely. In typical experimental situations, however, the system is supplied with energy to balance the energy dissipation [6 8,23. Theoretically, it is natural to consider white noise forcing 22,49, i.e., coupling to a thermal heat bath which leads to a nonequilibrium steady state. Interestingly, a stretched exponential behavior, $P(v) \propto \exp \left(-v^{3 / 2}\right)$, is found for the driven inelastic hard sphere gas [22.

Specifically, we assume that in addition to changes due to collisions, velocities may also change due to an external forcing: $\left.\frac{d v_{j}}{d t}\right|_{\text {heat }}=\xi_{j}$ with $j=1, \ldots, d$. We use standard uncorrelated white noise $\left\langle\xi_{i}(t) \xi_{j}\left(t^{\prime}\right)\right\rangle=2 D \delta_{i j} \delta\left(t-t^{\prime}\right)$ with a zero average $\left\langle\xi_{j}\right\rangle=0$. The rate equation for the temperature is modified by the additional source term $\frac{d}{d t} T+\lambda T^{3 / 2}=2 D$, and the system approaches a steady state, $T_{\infty}=(2 D / \lambda)^{2 / 3}$. The relaxation toward this state is exponential, $\left|T_{\infty}-T\right| \sim e^{- \text {const. } \times t}$.

Uncorrelated white noise forcing amounts to diffusion in velocity space. Therefore, Eq. (7) is modified as follows, $\frac{\partial}{\partial \tau} \rightarrow \frac{\partial}{\partial \tau}+D k^{2}$. In the steady state, the Fourier transform, $F_{\infty}(\mathbf{k}) \equiv \Psi(y)$ with $y=D k^{2}$, obeys

$$
(1+y) \Psi(y)=\langle\Psi(\xi y) \Psi(\eta y)\rangle .
$$

This equation is solved recursively by employing the cumulant expansion

$$
\Psi(y)=\exp \left[\sum_{n=1} \psi_{n}(-y)^{n}\right] .
$$

The cumulants $\kappa_{n}$, defined as

$$
F_{\infty}(\mathbf{k})=\exp \left[\sum_{m=1}^{\infty} \frac{\kappa_{m}(i k)^{m}}{m !}\right]
$$

are related to the coefficients $\psi_{n}$, viz. $\kappa_{n}=(2 n) ! D^{n} \psi_{n}$. Writing $1+y=\exp \left[\sum_{n \geq 1}(-y)^{n} / n\right]$, we recast Eq. (41) into

$$
1=\left\langle\exp \left[-\sum_{n=1}^{\infty}\left(\widetilde{\psi}_{n}-n^{-1}\right)(-y)^{n}\right]\right\rangle,
$$

with the auxiliary variables $\widetilde{\psi}_{n}=\psi_{n}\left(1-\xi^{n}-\eta^{n}\right)$. The desired cumulants $\psi_{n}$ are obtained by evaluating recursively the angular integrals of the auxiliary variables, $\left\langle\widetilde{\psi}_{n}\right\rangle$, and then using the identities $\psi_{n}=\left\langle\widetilde{\psi}_{n}\right\rangle / \lambda_{n}$. In one dimension, $\left\langle\mu^{n}\right\rangle=1$ and one immediately obtains $\left\langle\widetilde{\psi}_{n}\right\rangle=n^{-1}$, and consequently $n \psi_{n}=\left[1-\epsilon^{2 n}-(1-\epsilon)^{2 n}\right]^{-1}$ [39]. In higher dimensions, the quantities $\left\langle\widetilde{\psi}_{n}\right\rangle$ acquire nontrivial dependence on $n$, e.g., $\left\langle\widetilde{\psi}_{1}\right\rangle=1,\left\langle\widetilde{\psi}_{2}\right\rangle=\frac{1}{2}\left\langle\widetilde{\psi}_{1}^{2}\right\rangle$, and $\left\langle\widetilde{\psi}_{3}\right\rangle=\left\langle\widetilde{\psi}_{1} \widetilde{\psi}_{2}\right\rangle-\frac{1}{6}\left\langle\widetilde{\psi}_{1}^{3}\right\rangle$. The first few values for $\psi_{n}$ can be then evaluated. In particular, $\psi_{1}=1 / \lambda_{1}$ and $\psi_{2}=\left\langle(1-\xi-\eta)^{2}\right\rangle /\left(2 \lambda_{1}^{2} \lambda_{2}\right)$, from which one can determine explicit expressions:

$$
\begin{aligned}
& \psi_{1}=\frac{d}{2 \epsilon(1-\epsilon)}, \\
& \psi_{2}=\frac{3 d^{2}}{4(d+2)\left(1-\epsilon^{2}\right)-12(1-\epsilon)^{2}\left(1+\epsilon^{2}\right)} .
\end{aligned}
$$

Thus, the steady state distribution is not purely Maxwellian.

To probe velocity correlations or alternatively, deviations from a factorizing Maxwellian distribution, we consider the quantity $Q$, defined in Eq. (26). At the steady state, it is given by

$$
Q=\frac{\Psi^{\prime \prime}(0)}{\left[\Psi^{\prime}(0)\right]^{2}}-1 .
$$

In terms of the first two coefficients of the cumulant expansion, $Q=2 \psi_{2} / \psi_{1}^{2}$. Substituting the value of these coefficients yields

$$
Q=\frac{6 \epsilon^{2}(1-\epsilon)}{(d+2)(1+\epsilon)-3(1-\epsilon)\left(1+\epsilon^{2}\right)} .
$$

Note that for a fixed spatial dimension, this quantity is maximal in the completely inelastic case. For instance, $Q_{\max }=2 / 11$ in two dimensions and $Q_{\max }=2 / 15$ in three dimensions. These values are smaller by an order of magnitude or more than the corresponding values in the unforced case. Intuitively, one expects that white noise forcing randomizes the velocities of the particles. Indeed, velocity correlations are much less pronounced in this case, as seen in Figure 3. Additionally, velocity correlations diminish as the dimension increases. At large dimensions, velocity correlations vanish according to $Q \sim d^{-1}$, indicating that the velocity distribution becomes purely Maxwellian, $\Psi(y) \rightarrow \exp (-y / 2)$, when $d \rightarrow \infty$.

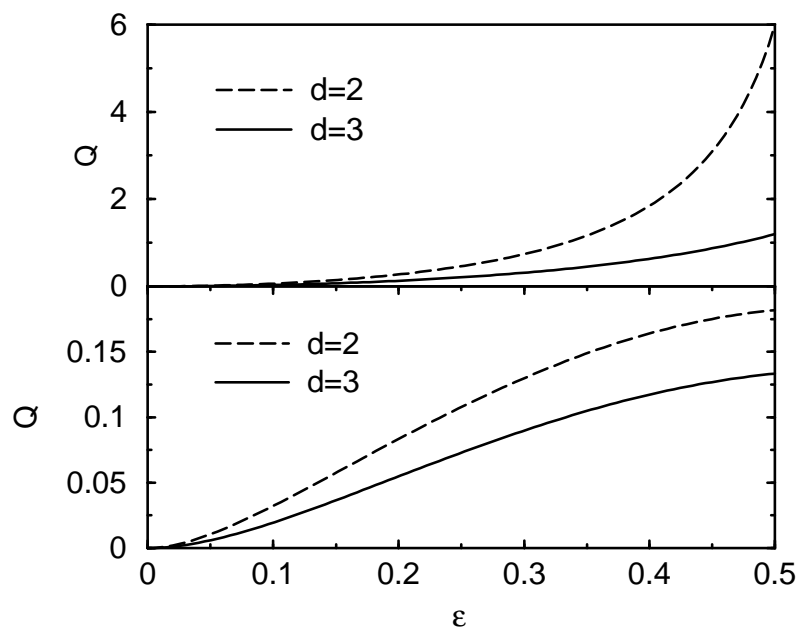

FIG. 3. The velocity correlation measure $Q$ versus the dissipation coefficient $\epsilon$. The scaling regime result 27) is shown in the top graph, and the steady state result (47) is shown in the bottom graph. 
The above results can be generalized in a number of ways. For example, the development of spatial correlations can be considered by placing particles on a lattice and allowing for nearest-neighbor collisions only. In this section we briefly mention two straightforward generalizations to: (1) energy generating collisions, and (2) distribution of restitution coefficients.

Thus far, we discussed only the physical case of dissipative collisions, namely $\epsilon<0$. However, the above results in the freely evolving case hold for energy generating collisions, i.e., $\epsilon>0$ as well. Although the typical velocity scale diverges, the velocity distribution still follows the scaling solution (11) with algebraic large velocity statistics. The corresponding exponent $\sigma$ is still obtained from Eq. (19). However, the behavior does change, as follows from the analytically tractable $d \rightarrow \infty$ behavior. In contrast with the dissipative case, the second term on the right-hand side of Eq. (19) now dominates, and it grows exponentially as $\propto a^{(\sigma-d) / 2}$. Since the left-hand side of Eq. (19) is of order unity, the constant $a$ must be equal to one. On the other hand, the constant $a$ is evaluated using the Stirling formula $\Gamma(x) \sim(x / e)^{x}$ to give $a=(1-\epsilon)^{2}(\sigma-d) d^{d /(\sigma-d)} \sigma^{-\sigma /(\sigma-d)}$. Equating $a=1$, we arrive at

$$
\sigma \cong d \nu, \quad \text { with } \quad(\nu-1) \nu^{-\frac{\nu}{\nu-1}}=(1-\epsilon)^{-2}
$$

While the exponent rises linearly with the dimension, it exhibit different $\epsilon$-dependence. Numerical solution of $\nu$ shows that this large dimension estimate again yields a useful approximation even at moderate dimensions.

Several recent studies have used a distribution of restitution coefficients to model driven granular systems, including for example, a one-dimensional gas of rods with internal degrees of freedom [50,51, and vertically vibrated layers [52]. By tuning the distribution properly, one can have a situation were overall, energy is conserved as dissipative collisions are balanced by energy generating collisions. When the restitution coefficient is drawn from the distribution $\rho(\epsilon)$, one simply integrates the collision integral in the Boltzmann equation (2) with respect to the measure $\rho(\epsilon)$. In one dimension, one can check that the scaling solution $\Phi(x)=(1+\sqrt{x}) e^{-\sqrt{x}}$ still holds, and in particular the exponent $\sigma=4$ is robust. In general dimension, the exponent $\sigma$ is given by

$$
1-\lambda \frac{\sigma-d}{2}=\int d \epsilon \rho(\epsilon)\left\langle\xi^{(\sigma-d) / 2}+\eta^{(\sigma-d) / 2}\right\rangle
$$

with the decay rate $\lambda=\int d \epsilon \rho(\epsilon) \lambda(\epsilon)$. We conclude that algebraic large-velocity statistics extend to situations where the dissipation coefficient $\epsilon$ is drawn from a given distribution.
We have studied inelastic gases within the framework of the Maxwell model, a Boltzmann equation with a uniform collision rate. We have shown that this kinetic theory is analytically tractable as closed evolution equations characterize the Fourier transform and consequently moments of the velocity distribution. In the freely evolving case, the system approaches a scaling regime, and the velocity distribution has an algebraic large velocity tail. The corresponding exponent varies continuously with the spatial dimension and the degree of dissipation. The decay exponents can be very large and therefore it may be difficult to distinguish a power law from a stretched exponential. In the driven case, we have determined the cumulants of the velocity distribution.

The time dependent behavior displays a number of interesting features. Moments of the velocity distribution exhibit multiscaling asymptotic behavior, and knowledge of the typical velocity is insufficient to characterize all moments. The velocity autocorrelation decays algebraically with time, and the corresponding exponent depends on the restitution coefficient only.

In contrast with elastic collisions, stochastic inelastic collision processes are not effective in mixing particle velocities. The stronger the inelasticity, the stronger the history dependence, i.e., memory of previous behavior. Additionally, inelasticity can generate significant correlations between different velocity components. Such correlations do develop even in the forced case, where dissipation is balanced by energy input, and one may expect that Maxwellian velocity distribution emerge.

The Maxwell model is truly mean field in nature with all aspects of the collision process being random. While it is not surprising that such a theory is solvable, the rich structure of the solution is somewhat unexpected. For example, the exponent follows from a transcendental equation, and can not be obtained from heuristic arguments or dimensional analysis. Remarkably, even the leading asymptotic behavior in the large dimension limit remains nontrivial as it involves roots of cubic or transcendental equations.

We have explored only the basic characteristics. Clearly, one can study higher order velocity correlation measures as well as higher order autocorrelations. Furthermore, the relaxation toward the steady state appears analytically tractable. The straightforward analysis is cumbersome and it may be useful to expand first the solutions in terms of more natural building blocks, e.g., orthogonal polynomials.

We stress that the Maxwell model is exact for stochastic inelastic collision processes with random collision partners and impact angles. It may be applicable in situations where an effective stirring mechanism leads to perfect mixing. Otherwise, it should be regarded as an uncontrolled approximation of the Boltzmann equation. Indeed, existing theoretical and numerical studies give 
little evidence for algebraic tails characterizing inelastic gases. The only exception was observed in a system with random restitution coefficients drawn from a broad distribution. In one dimension, both molecular dynamics simulation and direct integration of the Boltzmann equation for inelastic hard spheres show that the velocity distribution has a power law tail [52].

In conclusion, our results, combined with previous kinetic theory studies that find exponential, stretched exponential, and Gaussian tails, indicate that extremal velocity characteristics can be sensitive to the details of the model, let alone parameters such as the restitution coefficient, and the dimension.

We thank A. Baldassari and M. H. Ernst for fruitful correspondence, and $\mathrm{H}$. A. Rose for useful discussions. This research was supported by DOE (W-7405-ENG-36), NSF(DMR9978902), and ARO (DAAD19-99-1-0173).

[1] T. Pöschel and S. Luding (editors), Granular Gases (Springer, Berlin, 2000).

[2] H. J. Herrmann, in Disorder and Granular Media, edited by D. Bideau and A. Hansen (Elsevier Science Publishers, 1993).

[3] C. Bizon, M. D. Shattuck, J. B. Swift, W. D. McCormick, and H. L. Swinney, Phys. Rev. Lett. 80, 57 (1998).

[4] E. L. Grossman, T. Zhou, and E. Ben-Naim, Phys. Rev. E 55, 4200 (1997).

[5] A. Kudrolli, M. Wolpert, and J. P. Gollub, Phys. Rev. Lett. 78, 1383 (1997).

[6] W. Losert, D. G. W. Cooper, J. Delour, A. Kudrolli, and J. P. Gollub, Chaos 9, 682 (1999).

[7] J. S. Olafsen and J. S. Urbach, Phys. Rev. Lett. 81, 4369 (1998).

[8] X. Nie, E. Ben-Naim, and S. Y. Chen, Europhys. Lett. 51, 679 (2000).

[9] A. Samadani, L. Mahadevan, and A. Kudrolli, condmat/0110427.

[10] E. Rericha, C. Bizon, M. D. Shattuck, and H. L. Swinney, Phys. Rev. Lett. 88, 014302 (2002).

[11] J. T. Jenkins and M. W. Richman, Phys. Fluids 28, 3485 (1985).

[12] N. Sela and I. Goldhirsch, J. Fluid Mech. 361, 41 (1998).

[13] A. Goldshtein and M. Shapiro, J. Fluid Mech. 282, 75 (1995).

[14] P. K. Haff, J. Fluid Mech. 134, 401 (1983).

[15] G. F. Carnevale, Y. Pomeau, and W. R. Young, Phys. Rev. Lett. 64, 2913 (1990).

[16] B. Bernu and R. Mazighi, J. Phys. A 23, 5745 (1990).

[17] S. McNamara and W. R. Young, Phys. Fluids A 4, 496 (1992).

[18] Y. Du, H. Li, and L. P. Kadanoff, Phys. Rev. Lett. 74, 1268 (1995).

[19] T. Zhou and L. P. Kadanoff, Phys. Rev. E 54, 623 (1996).
[20] S. E. Esipov and T. Pöschel, J. Stat. Phys. 86, 1385 (1997).

[21] P. Deltour and A. Barrat, J. Phys. I France 7, 137 (1997).

[22] T. P. C. van Noije and M. H. Ernst, Granular Matter 1, 57 (1998).

[23] F. Rouyer and N. Menon, Phys. Rev. Lett. 85, 3676 (2000).

[24] I. S. Aranson and J. S. Olafsen, cond-mat/0110697.

[25] A. Puglisi, V. Loreto, U. Marini Bettolo Marconi, and A. Vulpiani, Phys. Rev. E 59, 5582 (1999).

[26] P. Résibois and M. de Leener, Classical Kinetic Theory of Fluids (John Wiley, New York, 1977).

[27] R. Soto and M. Mareschal, Phys. Rev. E 63, 041303 (2001).

[28] A. Barrat, T. Biben, Z. Rácz, E. Trizac, and F. van Wijland, J. Phys. A 35, 463 (2002).

[29] I. Goldhirsch and G. Zanetti, Phys. Rev. Lett. 70, 1619 (1993).

[30] R. Brito and M. H. Ernst, Europhys. Lett. 43, 497 (1998).

[31] E. Ben-Naim, S. Y. Chen, G. D. Doolen, and S. Redner, Phys. Rev. Lett. 83, 4069 (1999).

[32] I. Goldhirsch, in [1].

[33] J. C. Maxwell, Phil. Trans. R. Soc. 157, 49 (1867).

[34] C. Truesdell and R. G. Muncaster, Fundamentals of Maxwell's Kinetic Theory of a Simple Monoatomic Gas (Academic Press, New York, 1980).

[35] M. H. Ernst, Phys. Reports 78, 1 (1981).

[36] A. V. Bobylev, Sov. Sci. Rev. C. Math. Phys. 7, 111 (1988).

[37] L. Acedo, A. Santos, and A. V. Bobylev, condmat/0109490.

[38] H.-D. Kim and H. Hayakawa, cond-mat/0202003.

[39] E. Ben-Naim and P. L. Krapivsky, Phys. Rev. E 61, R5 (2000).

[40] A. Baldassarri, U. Marini Bettolo Marconi, and A. Puglisi, cond-mat/0111066.

[41] P. L. Krapivsky and E. Ben-Naim, J. Phys. A, in press.

[42] M. H. Ernst and R. Brito, cond-mat/0111093 and condmat/0112417.

[43] U. Marini Bettolo Marconi and A. Puglisi, condmat/0112336 and cond-mat/0202267.

[44] Every initial velocity distribution evolves towards the Maxwellian distribution if $d>1$ and $\epsilon=0$; for $d=1$, there is no relaxation in the elastic case.

[45] G. E. Andrews, R. Askey, and R. Roy, Special Functions (Cambridge University Press, New York, 1999).

[46] A. Baldassarri, private communication.

[47] N. V. Brilliantov and T. Pöschel, Phys. Rev. E 611716 (2000).

[48] H.-D. Kim and H. Hayakawa, J. Phys. Soc. Japan 70, 1954 (2001).

[49] J. A. Carrillo, C. Cercignani, and I. M. Gamba, Phys. Rev. E 62, 7700 (2000).

[50] G. Giese and A. Zippelius, Phys. Rev. E 54, 4828 (1996).

[51] T. Aspelmeier and A. Zippelius, Physica A 282, 450 (2000).

[52] A. Barrat, E. Trizac, and J. H. Fuchs, Eur. Phys. J. E 5, 161 (2001). 


\section{APPENDIX A: THE $\lambda$-COEFFICIENTS}

To compute the coefficients $\lambda_{n}=\left\langle 1-\xi^{n}-\eta^{n}\right\rangle$ and $\lambda_{n, m}=\left\langle\xi^{n} \eta^{m}\right\rangle$ we use $\xi=1-\left(1-\epsilon^{2}\right) \mu$ and $\eta=(1-\epsilon)^{2} \mu$. Thus, the following integrals are required

$$
\left\langle\mu^{n}\right\rangle=\frac{\Gamma\left(\frac{d}{2}\right) \Gamma\left(n+\frac{1}{2}\right)}{\Gamma\left(\frac{1}{2}\right) \Gamma\left(n+\frac{d}{2}\right)}=\frac{1}{d} \frac{3}{2+d} \cdots \frac{2(n-1)+1}{2(n-1)+d} .
$$

In particular, $\langle\mu\rangle=\frac{1}{d},\left\langle\mu^{2}\right\rangle=\frac{3}{d(d+2)},\left\langle\mu^{3}\right\rangle=\frac{15}{d(d+2)(d+4)}$, so the first few coefficients are

$$
\begin{aligned}
\lambda_{1}= & 2 \epsilon(1-\epsilon) \frac{1}{d} \\
\lambda_{2}= & 2\left(1-\epsilon^{2}\right) \frac{1}{d}-2(1-\epsilon)^{2}\left(1+\epsilon^{2}\right) \frac{3}{d(d+2)}, \\
\lambda_{3}= & 3\left(1-\epsilon^{2}\right) \frac{1}{d}-3\left(1-\epsilon^{2}\right)^{2} \frac{3}{d(d+2)} \\
& +2 \epsilon(1-\epsilon)^{3}\left(3+\epsilon^{2}\right) \frac{15}{d(d+2)(d+4)} \\
\lambda_{1,1}= & (1-\epsilon)^{2} \frac{1}{d}-(1-\epsilon)^{2}\left(1-\epsilon^{2}\right) \frac{3}{d(d+2)} .
\end{aligned}
$$

\section{APPENDIX B: THE AUTOCORRELATION EVOLUTION EQUATION}

It is useful to work with the collision counter $\tau$. In an infinitesimal time interval $\Delta \tau$, the velocity of a particle changes from $\mathbf{v} \equiv \mathbf{v}(\tau)$ to

$$
\mathbf{v}(\tau+\Delta \tau)= \begin{cases}\mathbf{v} & \text { prob. } 1-\Delta \tau \\ \mathbf{v}-(1-\epsilon)(\mathbf{v}-\mathbf{u}) \cdot \mathbf{n} \mathbf{n} & \text { prob. } \Delta \tau\end{cases}
$$

Here $\mathbf{u}$ is chosen randomly from all particles and the impact direction $\mathbf{n}$ is drawn from a uniform distribution. The rate of change in the autocorrelation function $A\left(\tau_{w}, \tau\right)=\overline{\mathbf{v}\left(\tau_{w}\right) \cdot \mathbf{v}(\tau)}$ is evaluated as follows

$$
\begin{aligned}
& \frac{d}{d \tau} A\left(\tau_{w}, \tau\right)=\lim _{\Delta \tau \rightarrow 0} \overline{\mathbf{v}\left(\tau_{w}\right) \cdot[\mathbf{v}(\tau+\Delta \tau)-\mathbf{v}(\tau)] / \Delta \tau} \\
& =-(1-\epsilon) \int d \mathbf{u} P(\mathbf{u}, \tau) \int d \mathbf{n} \overline{\left[\mathbf{v}\left(\tau_{w}\right) \cdot \mathbf{n}\right][(\mathbf{v}-\mathbf{u}) \cdot \mathbf{n}]} \\
& =-\frac{1-\epsilon}{d} \overline{\mathbf{v}\left(\tau_{w}\right) \cdot \mathbf{v}(\tau)}+\frac{1-\epsilon}{d} \int d \mathbf{u} P(\mathbf{u}, \tau) \overline{\mathbf{v}\left(\tau_{w}\right) \cdot \mathbf{u}} \\
& =-\frac{1-\epsilon}{d} A\left(\tau_{w}, \tau\right) .
\end{aligned}
$$

The angular integration in the second line of Eq. (B1) was performed using the identity

$$
H(\mathbf{a}, \mathbf{b})=\int d \mathbf{n}(\mathbf{a} \cdot \mathbf{n})(\mathbf{b} \cdot \mathbf{n})=\frac{1}{d}(\mathbf{a} \cdot \mathbf{b}) .
$$

This identity can be deduced by re-writing the integral as $H(\mathbf{a}, \mathbf{b})=\mathbf{a} \cdot \mathbf{h}(\mathbf{b})$. By symmetry, $\mathbf{h}(\mathbf{b})=\int d \mathbf{n} \mathbf{n}(\mathbf{b} \cdot \mathbf{n})$ is a vector along $\mathbf{b}$, say $\Lambda \mathbf{b}$, implying $H(\mathbf{a}, \mathbf{b})=\Lambda(\mathbf{a} \cdot \mathbf{b})$. Evaluating the special case $H(\mathbf{a}, \mathbf{a})=\langle\mu\rangle a^{2}$ we obtain (B2). Finally, the second term in the third line vanishes, $\overline{\mathbf{v}\left(\tau_{w}\right) \cdot \mathbf{u}(\tau)}=0$, since the velocity $\mathbf{u}(\tau)$ of the randomly chosen collision partner is uncorrelated with $\mathbf{v}\left(\tau_{w}\right)$. 